PROCEEDINGS OF THE

AMERICAN MATHEMATICAL SOCIETY

Volume 133, Number 2, Pages 397-401

S 0002-9939(04)07499-4

Article electronically published on August 30, 2004

\title{
THE BEST BOUNDS IN WALLIS' INEQUALITY
}

\author{
CHAO-PING CHEN AND FENG QI
}

(Communicated by Carmen C. Chicone)

AbStract. For all natural numbers $n$, let $n$ !! denote a double factorial. Then

$$
\frac{1}{\sqrt{\pi\left(n+\frac{4}{\pi}-1\right)}} \leq \frac{(2 n-1) ! !}{(2 n) ! !}<\frac{1}{\sqrt{\pi\left(n+\frac{1}{4}\right)}}
$$

The constants $\frac{4}{\pi}-1$ and $\frac{1}{4}$ are the best possible. From this, the well-known Wallis' inequality is improved.

\section{INTRODUCTION}

A double factorial $n$ !! can be defined by

$$
(2 m) ! !=\prod_{i=1}^{m}(2 i) \quad \text { and } \quad(2 m-1) ! !=\prod_{i=1}^{m}(2 i-1)
$$

for any given positive integer $m$. Let

$$
P_{n}=\frac{(2 n-1) ! !}{(2 n) ! !} .
$$

Then we have

(2) $\frac{1}{2 \sqrt{n}}<\frac{\sqrt{2}}{\sqrt{(2 n+1) \pi}}<P_{n}<\frac{2}{\sqrt{(4 n+1) \pi}}<\frac{1}{\sqrt{3 n+1}}<\frac{1}{\sqrt{2 n+1}}<\frac{1}{\sqrt{2 n}}$

for $n>1$. The inequality (2) is called Wallis' inequality in [18, p. 103].

The lower and upper bounds of $P_{n}$ in (21) are frequently cited and applied by mathematicians. The smallest upper bound $\frac{2}{\sqrt{(4 n+1) \pi}}$ and the largest lower bound $\frac{\sqrt{2}}{\sqrt{(2 n+1) \pi}}$ in (2)), that is, the inequalities

$$
\frac{\sqrt{2}}{\sqrt{(2 n+1) \pi}}<P_{n}<\frac{2}{\sqrt{(4 n+1) \pi}}
$$

Received by the editors August 3, 2002 and, in revised form, June 23, 2003 and September 27, 2003.

2000 Mathematics Subject Classification. Primary 05A10, 26D20; Secondary 33 B15.

Key words and phrases. Wallis' inequality, best bound, gamma function, monotonicity.

The authors were supported in part by NSF (\#10001016) of China, SF for the Prominent Youth of Henan Province (\#0112000200), SF of Henan Innovation Talents at Universities, NSF of Henan Province (\#004051800), Doctor Fund of Jiaozuo Institute of Technology, China. 
are obtained by N. D. Kazarinoff. See [16, pp. 47-48 and pp. 65-67]. We can rewrite inequality (3) as

$$
\frac{1}{\sqrt{\pi\left(n+\frac{1}{2}\right)}}<P_{n}<\frac{1}{\sqrt{\pi\left(n+\frac{1}{4}\right)}}
$$

for $n \in \mathbb{N}$.

The important use of formula (4) is to give a particular case of Wallis' formula (see [4, p. 259]) by taking $x=\frac{\pi}{2}$ in (ㅁ):

$$
\frac{\pi}{2}=\lim _{n \rightarrow \infty} \frac{[(2 n) ! !]^{2}}{[(2 n-1) ! !]^{2}(2 n+1)}=\prod_{n=1}^{\infty}\left[\frac{(2 n)^{2}}{(2 n-1)(2 n+1)}\right] .
$$

The Wallis formula follows originally from the infinite product representation of the sine (see [12, 23]):

$$
\sin x=x \prod_{n=1}^{\infty}\left(1-\frac{x^{2}}{\pi^{2} n^{2}}\right) .
$$

The Wallis formula can also be expressed as

$$
\frac{\pi}{2}=\left[4^{\zeta(0)} e^{-\zeta^{\prime}(0)}\right]^{2}
$$

see [12], where $\zeta$ is the Riemann zeta function [11].

A derivation of the Wallis formula from $\zeta^{\prime}(0)$ using the Hadamard product [10] for the Riemann zeta function $\zeta(s)$ due to Y. L. Yung can be found in [12]. The Wallis formula can also be reversed to derive $\zeta^{\prime}(0)$ from the Wallis formula without using the Hadamard product 22 .

It is noted that Wallis's sine (cosine) formula [13, 14] is as follows:

$$
\int_{0}^{\frac{\pi}{2}} \sin ^{n} x \mathrm{~d} x=\int_{0}^{\frac{\pi}{2}} \cos ^{n} x \mathrm{~d} x=\frac{\sqrt{\pi} \Gamma\left(\frac{n+1}{2}\right)}{n \Gamma\left(\frac{n}{2}\right)}= \begin{cases}\frac{\pi}{2} \cdot \frac{(n-1) ! !}{n ! !} & \text { for } n \text { even, } \\ \frac{(n-1) ! !}{n ! !} & \text { for } n \text { odd, }\end{cases}
$$

where $\Gamma$ is a gamma function.

An inequality involving the term $P_{n}$ is obtained by the second author and coworkers in [21] by using Tchebysheff's integral inequality.

It is well known that factorials and their "continuous" extension play an eminent role, for instance, in combinatorics, graph theory, and special functions.

For more information on the Wallis formula, please refer to [1, p. 258], [5, 6, 7], [8, pp. 17-28], [15, p. 468], [17, pp. 63-64], and the references therein.

In this article, we will refine inequality (44). More precisely, we will ask for two best possible constants $A$ and $B$ such that the double inequality

$$
\frac{1}{\sqrt{\pi(n+A)}} \leq P_{n} \leq \frac{1}{\sqrt{\pi(n+B)}}
$$

holds for all natural number $n$. In other words, the constants $A=\frac{4}{\pi}-1$ and $B=\frac{1}{4}$ cannot be replaced by smaller and larger numbers, respectively, in (9).

\section{LEMMAS}

Lemma 1. For $x>0$, we have

$$
\frac{2 x+1}{x(4 x+1)}<\frac{\Gamma^{\prime}\left(x+\frac{1}{2}\right)}{\Gamma\left(x+\frac{1}{2}\right)}-\frac{\Gamma^{\prime}(x)}{\Gamma(x)}
$$




$$
x^{b-a} \frac{\Gamma(x+a)}{\Gamma(x+b)}=1+\frac{(a-b)(a+b-1)}{2 x}+O\left(\frac{1}{x^{2}}\right), \quad x \rightarrow \infty .
$$

The proof of inequality (10) is given in [2, 3, 19, and the proof of the asymptotic expansion (11) can be found in [9] and [20, p. 378]. See also [1, p. 257].

Remark 1. Replacing $x$ by $x+\frac{1}{2}$ in (10) yields

$$
\frac{4 x+4}{(2 x+1)(4 x+3)}<\frac{\Gamma^{\prime}(x+1)}{\Gamma(x+1)}-\frac{\Gamma^{\prime}\left(x+\frac{1}{2}\right)}{\Gamma\left(x+\frac{1}{2}\right)} .
$$

Lemma 2. For $x>0$, we have

$$
\frac{\Gamma(x+1)}{\Gamma\left(x+\frac{1}{2}\right)}<\frac{2 x+1}{\sqrt{4 x+3}} .
$$

Proof. Define for a positive real number $x$,

$$
f(x)=\ln (2 x+1)-\frac{1}{2} \ln (4 x+3)-\ln \Gamma(x+1)+\ln \Gamma\left(x+\frac{1}{2}\right) .
$$

Differentiating $f(x)$ gives us

$$
f^{\prime}(x)=\frac{2}{2 x+1}-\frac{2}{4 x+3}-\left[\frac{\Gamma^{\prime}(x+1)}{\Gamma(x+1)}-\frac{\Gamma^{\prime}\left(x+\frac{1}{2}\right)}{\Gamma\left(x+\frac{1}{2}\right)}\right] .
$$

Utilizing (12), we obtain

$$
f^{\prime}(x)>\frac{2}{2 x+1}-\frac{2}{4 x+3}-\frac{4 x+4}{(2 x+1)(4 x+3)}=0 .
$$

Therefore, $f(x)$ is strictly increasing in $(0, \infty)$, and

$$
f(x)>f(0)=\frac{1}{2} \ln \frac{\pi}{3}>0,
$$

which leads to inequality (13).

Corollary 1. For all natural numbers n, we have

$$
\frac{\Gamma(n+1)}{\Gamma\left(n+\frac{1}{2}\right)}<\frac{2 n+1}{\sqrt{4 n+3}} .
$$

Corollary 2. The sequence

$$
\left\{Q_{n}\right\}_{n=1}^{\infty} \triangleq\left\{\left[\frac{\Gamma(n+1)}{\Gamma\left(n+\frac{1}{2}\right)}\right]^{2}-n\right\}_{n=1}^{\infty}
$$

is strictly decreasing.

Proof. The inequality $Q_{n+1}<Q_{n}$ is equivalent to

$$
\left[\frac{\Gamma(n+2)}{\Gamma\left(n+\frac{3}{2}\right)}\right]^{2}-(n+1)<\left[\frac{\Gamma(n+1)}{\Gamma\left(n+\frac{1}{2}\right)}\right]^{2}-n,
$$

which can be rewritten by using $\Gamma(x+1)=x \Gamma(x)$ as

$$
\begin{gathered}
{\left[\frac{n+1}{n+\frac{1}{2}} \cdot \frac{\Gamma(n+1)}{\Gamma\left(n+\frac{1}{2}\right)}\right]^{2}-1<\left[\frac{\Gamma(n+1)}{\Gamma\left(n+\frac{1}{2}\right)}\right]^{2},} \\
{\left[\frac{(n+1)^{2}}{\left(n+\frac{1}{2}\right)^{2}}-1\right]\left[\frac{\Gamma(n+1)}{\Gamma\left(n+\frac{1}{2}\right)}\right]^{2}<1,}
\end{gathered}
$$




$$
\begin{gathered}
{\left[\frac{\Gamma(n+1)}{\Gamma\left(n+\frac{1}{2}\right)}\right]^{2}<\frac{\left(n+\frac{1}{2}\right)^{2}}{(n+1)^{2}-\left(n+\frac{1}{2}\right)^{2}},} \\
\frac{\Gamma(n+1)}{\Gamma\left(n+\frac{1}{2}\right)}<\frac{2 n+1}{\sqrt{4 n+3}},
\end{gathered}
$$

which is a special case of inequality (14) by taking $x=n$. Therefore, the proof of monotonicity follows.

\section{MAin Results}

Now we give the main results of this paper.

Theorem 1. For all natural numbers n, we have

$$
\frac{1}{\sqrt{\pi\left(n+\frac{4}{\pi}-1\right)}} \leq \frac{(2 n-1) ! !}{(2 n) ! !}<\frac{1}{\sqrt{\pi\left(n+\frac{1}{4}\right)}} .
$$

The constants $\frac{4}{\pi}-1$ and $\frac{1}{4}$ are the best possible.

Proof. Since

$$
\Gamma(n+1)=n !, \quad \Gamma\left(n+\frac{1}{2}\right)=\frac{(2 n-1) ! !}{2^{n}} \sqrt{\pi}, \quad 2^{n} n !=(2 n) ! !,
$$

the double inequality (16) is equivalent to

$$
\frac{1}{4}<Q_{n}=\left[\frac{\Gamma(n+1)}{\Gamma\left(n+\frac{1}{2}\right)}\right]^{2}-n \leq \frac{4}{\pi}-1 .
$$

From the monotonicity of the sequence $Q_{n}$ provided in Corollary 2, it follows that

$$
\lim _{n \rightarrow \infty} Q_{n}<Q_{n} \leq Q_{1}=\frac{4}{\pi}-1 .
$$

Using the asymptotic formula (11), we conclude from

$$
Q_{n}=n\left[n^{-\frac{1}{2}} \frac{\Gamma(n+1)}{\Gamma\left(n+\frac{1}{2}\right)}-1\right]\left[n^{-\frac{1}{2}} \frac{\Gamma(n+1)}{\Gamma\left(n+\frac{1}{2}\right)}+1\right]
$$

that

$$
\lim _{n \rightarrow \infty} Q_{n}=\frac{1}{4} .
$$

Thus, inequality (17) follows. The proof is complete.

\section{ACKNOWLEDGEMENTS}

The authors are indebted to the anonymous referees and to the Editor, Professor Carmen Chicone, for their many valuable comments and corrections in language expressions. 


\section{REFERENCES}

[1] M. Abramowitz and I. A. Stegun (Eds.), Handbook of Mathematical Functions with Formulas, Graphs, and Mathematical Tables, National Bureau of Standards, Applied Mathematics Series 55, 9th printing, Dover, New York, 1972. MR 34:8607

[2] H. Alzer, On some inequalities for the gamma and psi functions, Math. Comp. 66 (1997), 373-389. MR 97e:33004

[3] G. D. Anderson, R. W. Barnard, K. C. Richards, M. K. Vamanamurthy, and M. Vuorinen, Inequalities for zero-balanced hypergeometric functions, Trans. Amer. Math. Soc. 347 (1995), 1713-1723. MR 95m:33002

[4] P. S. Bullen, A Dictionary of Inequalities, Pitman Monographs and Surveys in Pure and Applied Mathematics 97, Addison-Wesley, Longman Limited, 1998. MR 2000e:26001

[5] Ch.-P. Chen and F. Qi, Improvement of lower bound in Wallis' inequality, RGMIA Res. Rep. Coll. 5 (2002), suppl., Art. 23. Available online at http://rgmia.vu.edu.au/v5(E).html.

[6] Ch.-P. Chen and F. Qi, The best bounds in Wallis' inequality, RGMIA Res. Rep. Coll. 5 (2002), no. 4, Art. 13. Available online at http://rgmia.vu.edu.au/v5n4.html.

[7] Ch.-P. Chen and F. Qi, A new proof of the best bounds in Wallis' inequality, RGMIA Res. Rep. Coll. 6 (2003), no. 2, Art. 2. Available online at http://rgmia.vu.edu.au/v6n2.html.

[8] S. R. Finch, Archimedes' Constant, $\S 1.4$ in Mathematical Constants, Cambridge Univ. Press, Cambridge, England, 2003. Available online at http://pauillac.inria.fr/algo/bsolve/.

[9] C. L. Frenzer, Error bounds for asymptotic expansions of the ratio of two gamma functions, SIAM J. Math. Anal. 18 (1987), 890-896. MR 88d:33001

[10] http://mathworld.wolfram.com/HadamardProduct.html.

[11] http://mathworld.wolfram.com/RiemannZetaFunction.html.

[12] http://mathworld.wolfram.com/WallisFormula.html.

[13] http://mathworld.wolfram.com/WallisCosineFormula.html.

[14] http://mathworld.wolfram.com/WallisSineFormula.html.

[15] H. Jeffreys and B. S. Jeffreys, Wallis's Formula for $\pi$, $\S 15.07$ in Methods of Mathematical Physics, 3rd ed. Cambridge Univ. Press, Cambridge, England, 1988.

[16] N. D. Kazarinoff, Analytic Inequalities, Holt, Rhinehart and Winston, New York, 1961. MR 41:5577

[17] J. F. Kenney and E. S. Keeping, Mathematics of Statistics, Part 2, 2nd ed., Van Nostrand, Princeton, New Jersey, 1951.

[18] J.-Ch. Kuang, Chángyòng Bùděngshì (Applied Inequalities), 2nd edition, Hunan Education Press, Changsha, China, 1993. (Chinese) MR 95j:26001

[19] Y. L. Luke, Inequalities for the gamma function and its logarithmic derivative, Math. Balkanica (N. S.) 2 (1972), 118-123. MR 50:10338

[20] A. F. Nikiforov and V. B. Uvarov, Special Functions of Mathematical Physics, Birkhäuser, Basel, 1988. MR 89h:33001

[21] F. Qi, L.-H. Cui, and S.-L. Xu, Some inequalities constructed by Tchebysheff's integral inequality, Math. Inequal. Appl. 2 (1999), no. 4, 517-528. MR 2000m:26027

[22] J. Sondow, Analytic continuation of Riemann's zeta function and values at negative integers via Euler's transformation of series, Proc. Amer. Math. Soc. 120 (1994), 421-424. MR 94d:11066

[23] E. W. Weisstein, Concise Encyclopedia of Mathematics CD-ROM, CD-ROM edition 1.0, May 20, 1999. Available online at http://www.math.pku.edu.cn/stu/eresource/wsxy/sxrjjc/wk/ Encyclopedia/math/w/w009.htm

Department of Applied Mathematics and Informatics, Research Institute of Applied Mathematics, Henan Polytechnic University, Jiaozuo City, Henan 454000, People's RePUBLIC OF CHINA

E-mail address: chenchaoping@hpu.edu.cn

Department of Applied Mathematics and Informatics, Research Institute of Applied Mathematics, Henan Polytechnic University, Jiaozuo City, Henan 454000, People's RePUBLIC OF CHINA

E-mail address: qifeng@hpu.edu.cn

E-mail address: fengqi618@member.ams.org

$U R L:$ http://rgmia.vu.edu.au/qi.html 